\title{
Existence results of positive solutions for boundary value problems of fractional differential equations
}

\section{Guoqing Chai*}

\section{"Correspondence:}

mathchgq@gmail.com

College of Mathematics and

Statistics, Hubei Normal University,

Hubei, 435002, P.R. China

\section{Abstract}

In this paper, we are concerned with the following fractional equation:

$$
{ }^{C} D_{0+}^{\alpha} u(t)=f\left(t, u(t), u^{\prime}(t)\right), \quad t \in(0,1)
$$

with the boundary value conditions

$$
u(1)=u^{\prime}(1)=0, \quad \delta u^{\prime \prime}(0)=u^{\prime \prime}(1), \quad \gamma u^{\prime \prime \prime}(0)=u^{\prime \prime \prime}(1),
$$

where ${ }^{C} D_{0+}^{\alpha}$ is the standard Caputo derivative with $3<\alpha \leq 4$ and $\delta, \gamma$ are constants with $\delta>1, \gamma>1$. By applying a new fixed point theorem on cone and Krasnoselskii's fixed point theorem, some existence results of positive solution are obtained.

MSC: $34 \mathrm{~A} 08 ; 34 \mathrm{~B} 15 ; 34 \mathrm{~B} 18$

Keywords: fractional differential equations; existence results; fixed point theorem; positive solution

\section{Introduction}

In this paper, we are concerned with the existence of positive solutions for the fractional equation

$$
{ }^{C} D_{0_{+}}^{\alpha} u(t)=f\left(t, u(t), u^{\prime}(t)\right), \quad t \in(0,1)
$$

with the boundary value conditions

$$
u(1)=u^{\prime}(1)=0, \quad \delta u^{\prime \prime}(0)=u^{\prime \prime}(1), \quad \gamma u^{\prime \prime \prime}(0)=u^{\prime \prime \prime}(1),
$$

where ${ }^{C} D_{0+}^{\alpha}$ is the standard Caputo derivative with $3<\alpha \leq 4$ and $\delta, \gamma$ are constants with $\delta>1, \gamma>1$.

Differential equations of fractional order have recently proved to be valuable tools in the modeling of many phenomena in various fields of science and engineering. Indeed, we can find numerous applications in viscoelasticity, electrochemistry, control, porous media, electromagnetism, etc. (see [1-5]). There has been a significant development in the study of fractional differential equations and inclusions in recent years, see the monographs of Podlubny [5], Kilbas et al. [6], Lakshmikantham et al. [7], Samko et al. [8], Diethelm [9], 
and the survey by Agarwal et al. [10]. For some recent contributions on fractional differential equations, see [9-25] and the references therein.

On the other hand, it is well known that the fourth-order boundary value problem describes the deformations of an elastic beam in equilibrium state. Owing to its importance in physics, the existence of solutions to this problem has been studied by many authors; see, for example, [26-30] and references therein. Recently, there have been a few papers dealing with the existence of solutions for fractional equations of order $\alpha \in(3,4]$.

In [14], Xu et al. discussed the problem

$$
D_{0+}^{\alpha} u(t)=f(t, u(t)), \quad t \in(0,1), u(0)=u^{\prime}(0)=u(1)=u^{\prime}(1)=0,
$$

where $3<\alpha \leq 4$ and $f \in C([0,1] \times(0, \infty))$ is nonnegative, $D_{0_{+}}^{\alpha}$ is the Riemann-Liouville fractional derivative of order $\alpha$. The existence results of positive solutions are obtained by applying the Leray-Schauder nonlinear alternative theorem.

In [15], Liang and Zhang studied the following nonlinear fractional boundary value problem:

$$
D_{0+}^{\alpha} u(t)=f(t, u(t)), \quad t \in(0,1), u(0)=u^{\prime}(0)=u^{\prime \prime}(0)=u^{\prime \prime}(1)=0,
$$

where $3<\alpha \leq 4, f(t, u) \in C([0,1] \times[0, \infty),[0, \infty))$ is nondecreasing relative to $u, D_{0_{+}}^{\alpha}$ is the Riemann-Liouville fractional derivative of order $\alpha$. By means of the lower and upper solution method and fixed point theorems, some results on the existence of positive solutions were obtained.

In [16], Agarwal and Ahmad studied the solvability of the following anti-periodic boundary value problem for a nonlinear fractional differential equation:

$$
\begin{cases}D_{0+}^{\alpha} u(t)=f(t, u(t)), & t \in(0, T), \\ u(0)+u(T)=0, & u^{\prime}(0)+u^{\prime}(T)=0, \\ u^{\prime \prime}(0)+u^{\prime \prime}(T)=0, & u^{\prime \prime \prime}(0)+u^{\prime \prime \prime}(T)=0\end{cases}
$$

where $3<\alpha \leq 4$. The existence results were obtained by the nonlinear alternative theorem.

Inspired by above work, the author will be concerned with the boundary value problem (BVP for short in the sequel) (1.1)-(1.2). To the best of our knowledge, no contribution exists concerning the existence of solutions for BVP (1.1)-(1.2). In the present paper, by applying a new fixed point theorem on cone and Krasnoselskii's fixed point theorem, some existence results of positive solution for BVP (1.1)-(1.2) are obtained. It is worth to point out that the results in this paper are also new even for $\alpha=4$ relative to the corresponding literature with regard to the fourth-order boundary value problem. In addition, the conditions imposed in this paper are easily verified.

The organization of this paper is as follows. In Section 2, we present some necessary definitions and preliminary results that will be used to prove our main results. In Section 3, we put forward and prove our main results. Finally, we give two examples to demonstrate our main results.

\section{Preliminaries}

In this section, we introduce some preliminary facts which are useful throughout this paper. 
Let $\mathbb{N}$ be the set of positive integers, $\mathbb{R}$ be the set of real numbers, $\mathbb{R}_{+}=[0,+\infty)$, and $\mathbb{R}_{-}=(-\infty, 0]$. Let $I=[0,1]$. Denote by $C^{1}(I, \mathbb{R})$ the Banach space endowed with the norm $\|u\|_{1}=\|u\|_{0}+\left\|u^{\prime}\right\|_{0}$, where $\|u\|_{0}=\max _{t \in I}|u(t)|$ for $u \in C(I, \mathbb{R})$.

Definition 2.1 [6] The Riemann-Liouville fractional integral of order $\alpha>0$ of a function $y:(a, b] \rightarrow \mathbb{R}$ is given by

$$
I_{a+}^{\alpha} y(t)=\frac{1}{\Gamma(\alpha)} \int_{0}^{t}(t-s)^{\alpha-1} y(s) d s, \quad t \in(a, b] .
$$

Definition 2.2 [6] The Riemann-Liouville fractional derivative of order $\alpha>0$ of a function $y:(a, b] \rightarrow \mathbb{R}$ is given by

$$
D_{a+}^{\alpha} y(t)=\frac{1}{\Gamma(n-\alpha)}\left(\frac{d}{d t}\right)^{n} \int_{0}^{t} \frac{y(s)}{(t-s)^{\alpha-n+1}} d s, \quad t \in(a, b],
$$

where $n=[\alpha]+1,[\alpha]$ denotes the integer part of $\alpha$.

Definition 2.3 [6] The Caputo fractional derivative of order $\alpha>0$ of a function $y$ on $(a, b$ ] is defined via the above Riemann-Liouville derivatives by

$$
\left({ }^{C} D_{a+}^{\alpha} y\right)(x)=\left(D_{a+}^{\alpha}\left[y(t)-\sum_{k=0}^{n-1} \frac{y^{(k)}(a)}{k !}(t-a)^{k}\right]\right)(x), \quad x \in(a, b] .
$$

Lemma 2.1 [6] Let $\alpha>0$ and $y \in C[a, b]$. Then

$$
\left({ }^{C} D_{a+}^{\alpha} I_{a+}^{\alpha} y\right)(x)=y(x)
$$

holds on $[a, b]$.

Lemma 2.2 [17] Let $n \in N$ with $n \geq 2, \alpha \in(n-1, n]$. If $y \in C^{n-1}[a, b]$ and ${ }^{C} D_{a+}^{\alpha} y \in C(a, b)$, then

$$
I_{a+}^{\alpha{ }^{C}} D_{a+}^{\alpha} y(t)=y(t)-\sum_{k=0}^{n-1} \frac{y^{(k)}(a)}{k !}(t-a)^{k}
$$

holds on $(a, b)$.

For convenience, we first list some hypotheses which will be used throughout this paper.

$\left(H_{1}\right) f \in C\left(I \times \mathbb{R}_{+} \times \mathbb{R}_{-}, \mathbb{R}_{+}\right)$.

$\left(H_{2}\right) 3<\alpha \leq 4, \gamma>1, \delta>1$.

For $h \in C[0,1]$, consider the following BVP:

$$
\begin{aligned}
& { }^{C} D_{0+}^{\alpha} u(t)=h(t), \quad t \in(0,1), \\
& u(1)=u^{\prime}(1)=0, \quad \delta u^{\prime \prime}(0)=u^{\prime \prime}(1), \quad \gamma u^{\prime \prime \prime}(0)=u^{\prime \prime \prime}(1) .
\end{aligned}
$$

We have the following lemma, which is important in this paper. 
Lemma 2.3 Let $\left(H_{2}\right)$ hold. Then $u \in C^{3}[0,1]$ is a solution of $B V P(2.1)-(2.2)$ iff $u \in C[0,1]$ has the expression as follows:

$$
u(t)=\int_{0}^{1} G(t, s) h(s) d s, \quad t \in I
$$

where

$$
G(t, s)= \begin{cases}G_{1}(t, s), & 0 \leq s \leq t<1 \\ G_{2}(t, s), & 0 \leq t \leq s<1\end{cases}
$$

and

$$
\begin{aligned}
G_{1}(t, s)= & \frac{\left[(t-s)^{\alpha-1}-(1-s)^{\alpha-1}\right]}{\Gamma(\alpha)}+\frac{(1-t)(1-s)^{\alpha-2}}{\Gamma(\alpha-1)} \\
& +\frac{(1-t)^{2}(1-s)^{\alpha-3}}{2(\delta-1) \Gamma(\alpha-2)}+\frac{(1-t)^{2}[1+2 \delta+(\delta-1) t]}{6(\gamma-1)(\delta-1) \Gamma(\alpha-3)}(1-s)^{\alpha-4}, \\
G_{2}(t, s)= & -\frac{(1-s)^{\alpha-1}}{\Gamma(\alpha)}+\frac{(1-t)(1-s)^{\alpha-2}}{\Gamma(\alpha-1)}+\frac{(1-t)^{2}(1-s)^{\alpha-3}}{2(\delta-1) \Gamma(\alpha-2)} \\
& +\frac{(1-t)^{2}[1+2 \delta+(\delta-1) t]}{6(\gamma-1)(\delta-1) \Gamma(\alpha-3)}(1-s)^{\alpha-4}
\end{aligned}
$$

Proof Let $u \in C^{3}[0,1]$ be a solution of (2.1)-(2.2). Then by Lemma 2.2, we have

$$
u(t)=c_{0}+c_{1} t+c_{2} t^{2}+c_{3} t^{3}+I_{0+}^{\alpha} h(t), \quad t \in I,
$$

and so

$$
\begin{aligned}
& u^{\prime}(t)=c_{1}+2 c_{2} t+3 c_{3} t^{2}+I_{0+}^{\alpha-1} h(t), \quad t \in I, \\
& u^{\prime \prime}(t)=2 c_{2}+6 c_{3} t+I_{0+}^{\alpha-2} h(t), \quad t \in I, \\
& u^{\prime \prime \prime}(t)=6 c_{3}+I_{0+}^{\alpha-3} h(t), \quad t \in I .
\end{aligned}
$$

Thus, by the boundary value condition (2.2), we can obtain

$$
\begin{aligned}
& c_{0}+c_{1}+c_{2}+c_{3}+I_{0+}^{\alpha} h(1)=0, \\
& c_{1}+2 c_{2}+3 c_{3}+I_{0+}^{\alpha-1} h(1)=0, \\
& 2(1-\delta) c_{2}+6 c_{3}+I_{0+}^{\alpha-2} h(1)=0, \\
& 6(1-\gamma) c_{3}+I_{0+}^{\alpha-3} h(1)=0 .
\end{aligned}
$$

From (2.11), we have

$$
c_{3}=\frac{1}{6(\gamma-1)} I_{0+}^{\alpha-3} h(1)
$$

Substituting (2.12) into (2.10), we get

$$
c_{2}=\frac{1}{2(\gamma-1)(\delta-1)} I_{0+}^{\alpha-3} h(1)+\frac{1}{2(\delta-1)} I_{0+}^{\alpha-2} h(1) .
$$


So, by (2.13), (2.12), and (2.9), we have

$$
c_{1}=-\frac{1+\delta}{2(\gamma-1)(\delta-1)} I_{0+}^{\alpha-3} h(1)-\frac{1}{\delta-1} I_{0+}^{\alpha-2} h(1)-I_{0+}^{\alpha-1} h(1) .
$$

Thus, from (2.8), we have

$$
c_{0}=\frac{1+2 \delta}{6(\gamma-1)(\delta-1)} I_{0+}^{\alpha-3} h(1)+\frac{1}{2(\delta-1)} I_{0+}^{\alpha-2} h(1)+I_{0+}^{\alpha-1} h(1)-I_{0+}^{\alpha} h(1) .
$$

Hence, from (2.7) together with (2.12)-(2.15), it follows that

$$
\begin{aligned}
u(t)= & c_{0}+c_{1} t+c_{2} t^{2}+c_{3} t^{3}+I_{0+}^{\alpha} h(t) \\
= & -I_{0+}^{\alpha} h(1)+(1-t) I_{0+}^{\alpha-1} h(1)+\frac{1}{2(\delta-1)}(1-t)^{2} I_{0+}^{\alpha-2} h(1) \\
& +\frac{\left[(2 \delta+1)-3(1+\delta) t+3 t^{2}+(\delta-1) t^{3}\right]}{6(\gamma-1)(\delta-1)} I_{0+}^{\alpha-3} h(1)+I_{0+}^{\alpha} h(t) .
\end{aligned}
$$

Noticing that

$$
(2 \delta+1)-3(1+\delta) t+3 t^{2}+(\delta-1) t^{3}=(1-t)^{2}[1+2 \delta+(\delta-1) t]
$$

by Definition 2.1, we have

$$
\begin{aligned}
u(t)= & \int_{0}^{t}\left[\frac{(t-s)^{\alpha-1}-(1-s)^{\alpha-1}}{\Gamma(\alpha)}+\frac{(1-t)(1-s)^{\alpha-2}}{\Gamma(\alpha-1)}+\frac{(1-t)^{2}(1-s)^{\alpha-3}}{2(\delta-1) \Gamma(\alpha-2)}\right. \\
& \left.+\frac{(1-t)^{2}[1+2 \delta+(\delta-1) t]}{6(\gamma-1)(\delta-1) \Gamma(\alpha-3)}(1-s)^{\alpha-4}\right] h(s) d s \\
& +\int_{t}^{1}\left[-\frac{(1-s)^{\alpha-1}}{\Gamma(\alpha)}+\frac{(1-t)(1-s)^{\alpha-2}}{\Gamma(\alpha-1)}+\frac{(1-t)^{2}(1-s)^{\alpha-3}}{2(\delta-1) \Gamma(\alpha-2)}\right. \\
& \left.+\frac{(1-t)^{2}[1+2 \delta+(\delta-1) t]}{6(\gamma-1)(\delta-1) \Gamma(\alpha-3)}(1-s)^{\alpha-4}\right] h(s) d s \\
= & \int_{0}^{1} G(t, s) h(s) d s, \quad t \in I .
\end{aligned}
$$

Conversely, if $u$ has the expression (2.3), then from the fact that $h \in C[0,1]$, we can easily verify that

$$
\begin{aligned}
u^{\prime}(t)= & \int_{0}^{t}\left[\frac{(t-s)^{\alpha-2}-(1-s)^{\alpha-2}}{\Gamma(\alpha-1)}+\frac{(t-1)(1-s)^{\alpha-3}}{(\delta-1) \Gamma(\alpha-2)}+\frac{(t-1)[1+\delta+(\delta-1) t]}{2(\gamma-1)(\delta-1) \Gamma(\alpha-3)}\right] h(s) d s \\
& +\int_{t}^{1}\left[-\frac{(1-s)^{\alpha-2}}{\Gamma(\alpha-1)}+\frac{(t-1)(1-s)^{\alpha-3}}{(\delta-1) \Gamma(\alpha-2)}+\frac{(t-1)[1+\delta+(\delta-1) t]}{2(\gamma-1)(\delta-1) \Gamma(\alpha-3)}\right] h(s) d s, \\
u^{\prime \prime}(t)= & \int_{0}^{t}\left[\frac{(t-s)^{\alpha-3}}{\Gamma(\alpha-2)}+\frac{(1-s)^{\alpha-3}}{(\delta-1) \Gamma(\alpha-2)}+\frac{1+(\delta-1) t}{(\gamma-1)(\delta-1) \Gamma(\alpha-3)}\right] h(s) d s \\
& +\int_{t}^{1}\left[\frac{(1-s)^{\alpha-3}}{(\delta-1) \Gamma(\alpha-2)}+\frac{1+(\delta-1) t}{(\gamma-1)(\delta-1) \Gamma(\alpha-3)}\right] h(s) d s,
\end{aligned}
$$




$$
u^{\prime \prime \prime}(t)=\int_{0}^{t}\left[\frac{(t-s)^{\alpha-4}}{\Gamma(\alpha-3)}+\frac{1}{(\gamma-1) \Gamma(\alpha-3)}\right] h(s) d s+\int_{t}^{1} \frac{1}{(\gamma-1) \Gamma(\alpha-3)} h(s) d s
$$

hold for $t \in I$, and $u$ satisfies the boundary condition (2.2).

Again, from (2.16) and Lemma 2.1, we have that ${ }^{C} D_{0+}^{\alpha} x(t)=h(t), t \in(0,1)$. In addition, noting that $h \in C[0,1]$, it is easy to see that $x \in C^{3}[0,1]$ from $(2.19)$.

For the forthcoming analysis, we need to introduce some new notations.

Let $\eta_{1}=\min \left\{\frac{2 \alpha-5}{3(\alpha-1)}, \frac{4(1+2 \delta)}{27 \delta}\right\}$, and $\eta_{2}=\min \left\{\frac{2(\alpha-2)}{3(\alpha+\delta-3)}, \frac{1+\delta}{3 \delta}\right\}$. Denote

$$
\begin{aligned}
& w_{1}(s)=\frac{(1-s)^{\alpha-2}}{\Gamma(\alpha-1)}+\frac{(1-s)^{\alpha-3}}{2(\delta-1) \Gamma(\alpha-2)}+\frac{\delta(1-s)^{\alpha-4}}{2(\gamma-1)(\delta-1) \Gamma(\alpha-3)}, \quad s \in[0,1), \\
& w_{2}(s)=\frac{(\alpha+\delta-3)(1-s)^{\alpha-3}}{(\alpha-2)(\delta-1) \Gamma(\alpha-2)}+\frac{\delta(1-s)^{\alpha-4}}{(\gamma-1)(\delta-1) \Gamma(\alpha-3)}, \quad s \in[0,1) .
\end{aligned}
$$

It is easy to verify that $\eta_{1}, \eta_{2} \in(0,1)$, and $w_{1}, w_{2} \in C([0,1),(0,+\infty))$ noting that $3<\alpha \leq 4$, $\gamma>1, \delta>1$.

We also need the following lemma, which will play an important role in obtaining our main results in Section 3.

Lemma 2.4 Under the assumption $\left(H_{2}\right)$, Green's function $G(t, s)$ has the following properties:

(1) $G$ is continuous on $[0,1) \times[0,1)$;

(2) $G(t, s)>0, t, s \in[0,1) ; G_{t}(t, s)<0, t, s \in[0,1), s \neq t$;

(3) $G(t, s) \leq w_{1}(s), t, s \in[0,1) ; G(t, s) \geq \eta_{1} w_{1}(s), t \in\left[\frac{1}{6}, \frac{1}{3}\right], s \in[0,1)$;

$0<-G_{t}(t, s) \leq w_{2}(s), t, s \in[0,1), s \neq t ;-G_{t}(t, s) \geq \eta_{2} w_{2}(s), t \in\left[\frac{1}{6}, \frac{1}{3}\right], s \in[0,1), s \neq t$.

Proof (1) Observing the expression of Green's function given by (2.4)-(2.6), the conclusion (1) of Lemma 2.4 is obvious.

(2) We first show that $G_{t}(t, s)<0, t, s \in[0,1), s \neq t$.

In fact, if $0 \leq s<t<1$, then by (2.5) we have

$$
\begin{aligned}
\frac{\partial G_{1}}{\partial t}= & \frac{\left[(t-s)^{\alpha-2}-(1-s)^{\alpha-2}\right]}{\Gamma(\alpha-1)}-\frac{(1-t)(1-s)^{\alpha-3}}{(\delta-1) \Gamma(\alpha-2)} \\
& -\frac{(1-t)[1+\delta+(\delta-1) t]}{2(\gamma-1)(\delta-1) \Gamma(\alpha-3)}(1-s)^{\alpha-4} .
\end{aligned}
$$

Owing to the fact that $3<\alpha \leq 4$ and $0 \leq s<t<1$, we have that $(t-s)^{\alpha-2}<(1-s)^{\alpha-2}$. Thus, we immediately obtain that $\frac{\partial G_{1}}{\partial t}<0$ for $0 \leq s<t<1$ from (2.20) together with the condition $\delta>1, \gamma>1$.

Similarly, we can deduce that

$$
\frac{\partial G_{2}}{\partial t}=-\frac{(1-s)^{\alpha-2}}{\Gamma(\alpha-1)}-\frac{(1-t)(1-s)^{\alpha-3}}{(\delta-1) \Gamma(\alpha-2)}-\frac{(1-t)[1+\delta+(\delta-1) t]}{2(\gamma-1)(\delta-1) \Gamma(\alpha-3)}(1-s)^{\alpha-4}
$$

for $0 \leq t<s<1$, and so $\frac{\partial G_{2}}{\partial t}<0$ for $0 \leq t<s<1$.

To summarize, $\frac{\partial G}{\partial t}<0$ for all $s, t \in[0,1)$ with $s \neq t$. 
Now, since $G(1, s)=G_{1}(1, s)=0$ for $0 \leq s<1$, and $\frac{\partial G}{\partial t}<0$ for $t, s \in[0,1)$ with $t \neq s$, it follows that $G(t, s)>0$ for all $t, s \in[0,1)$.

(3) The proof is divided into four steps.

Step 1 . We show that $G(t, s) \leq w_{1}(s)$ for $t, s \in[0,1)$.

(i) If $0 \leq s \leq t<1$, then by (2.5) and the assumption that $3<\alpha \leq 4, \gamma>1$, and $\delta>1$, we have

$$
\begin{aligned}
G_{1}(t, s) & \leq \frac{(1-t)(1-s)^{\alpha-2}}{\Gamma(\alpha-1)}+\frac{(1-t)^{2}(1-s)^{\alpha-3}}{2(\delta-1) \Gamma(\alpha-2)}+\frac{(1-t)^{2}[1+2 \delta+(\delta-1) t]}{6(\gamma-1)(\delta-1) \Gamma(\alpha-3)}(1-s)^{\alpha-4} \\
& <\frac{(1-s)^{\alpha-2}}{\Gamma(\alpha-1)}+\frac{(1-s)^{\alpha-3}}{2(\delta-1) \Gamma(\alpha-2)}+\frac{\delta(1-s)^{\alpha-4}}{2(\gamma-1)(\delta-1) \Gamma(\alpha-3)} \triangleq w_{1}(s)
\end{aligned}
$$

(ii) If $0 \leq t \leq s<1$, then by an argument similar to (2.22), we have

$$
G_{2}(t, s) \leq w_{1}(s)
$$

Summing up the above analysis (i)-(ii), we obtain

$$
G(t, s) \leq w_{1}(s) \text { for } t, s \in[0,1)
$$

Step 2. We show that $G(t, s) \geq \eta_{1} w_{1}(s)$ for $t \in\left[\frac{1}{6}, \frac{1}{3}\right]$ and $s \in[0,1)$.

In fact, if $0 \leq s \leq t$ and $t \in\left[\frac{1}{6}, \frac{1}{3}\right]$, then by (2.5) combined with the assumption that $3<\alpha \leq 4, \gamma>1, \delta>1$, we have

$$
\begin{aligned}
G_{1}(t, s) \geq & -\frac{(1-s)^{\alpha-1}}{\Gamma(\alpha)}+\frac{(1-t)(1-s)^{\alpha-2}}{\Gamma(\alpha-1)}+\frac{(1-t)^{2}(1-s)^{\alpha-3}}{2(\delta-1) \Gamma(\alpha-2)} \\
& +\frac{(1-t)^{2}[1+2 \delta+(\delta-1) t]}{6(\gamma-1)(\delta-1) \Gamma(\alpha-3)}(1-s)^{\alpha-4} \\
\geq & -\frac{(1-s)^{\alpha-2}}{\Gamma(\alpha)}+\frac{2(1-s)^{\alpha-2}}{3 \Gamma(\alpha-1)}+\frac{2(1-s)^{\alpha-3}}{9(\delta-1) \Gamma(\alpha-2)}+\frac{2(1+2 \delta)(1-s)^{\alpha-4}}{27(\gamma-1)(\delta-1) \Gamma(\alpha-3)} \\
= & \frac{(2 \alpha-5)}{3(\alpha-1)} \cdot \frac{(1-s)^{\alpha-2}}{\Gamma(\alpha-1)}+\frac{4}{9} \cdot \frac{(1-s)^{\alpha-3}}{2(\delta-1) \Gamma(\alpha-2)} \\
& +\frac{4(1+2 \delta)}{27 \delta} \cdot \frac{\delta(1-s)^{\alpha-4}}{2(\gamma-1)(\delta-1) \Gamma(\alpha-3)} \geq \eta_{1} w_{1}(s),
\end{aligned}
$$

where $\eta_{1}=\min \left\{\frac{2 \alpha-5}{3(\alpha-1)}, \frac{4}{9}, \frac{4(1+2 \delta)}{27 \delta}\right\}=\min \left\{\frac{2 \alpha-5}{3(\alpha-1)}, \frac{4(1+2 \delta)}{27 \delta}\right\}$, because $\frac{2 \alpha-5}{3(\alpha-1)}<\frac{4}{9}$.

If $t \in\left[\frac{1}{6}, \frac{1}{3}\right]$ with $t \leq s<1$, then by an argument similar to (2.23), we have

$$
G_{2}(t, s) \geq \eta_{1} w_{1}(s)
$$

So, by (2.23)-(2.24), we have

$G(t, s) \geq \eta_{1} w_{1}(s)$ for $s \in[0,1)$, and $t \in\left[\frac{1}{6}, \frac{1}{3}\right]$.

Step 3. Now, we show that

$$
0<-\frac{\partial G(t, s)}{\partial t} \leq w_{2}(s) \text { for } s, t \in[0,1), s \neq t
$$


(i) If $0 \leq s<t<1$, then by (2.20) and keeping in mind that $3<\alpha \leq 4, \gamma>1, \delta>1$, it follows that

$$
\begin{aligned}
-\frac{\partial G_{1}}{\partial t}= & -\frac{(t-s)^{\alpha-2}}{\Gamma(\alpha-1)}+\frac{(1-s)^{\alpha-2}}{\Gamma(\alpha-1)}+\frac{(1-t)(1-s)^{\alpha-3}}{(\delta-1) \Gamma(\alpha-2)} \\
& +\frac{(1-t)[1+\delta+(\delta-1) t]}{2(\gamma-1)(\delta-1) \Gamma(\alpha-3)}(1-s)^{\alpha-4} \\
\leq & \frac{(1-s)^{\alpha-2}}{\Gamma(\alpha-1)}+\frac{(1-s)^{\alpha-3}}{(\delta-1) \Gamma(\alpha-2)}+\frac{\delta(1-s)^{\alpha-4}}{(\gamma-1)(\delta-1) \Gamma(\alpha-3)} \\
\leq & \frac{(1-s)^{\alpha-3}}{\Gamma(\alpha-1)}+\frac{(1-s)^{\alpha-3}}{(\delta-1) \Gamma(\alpha-2)}+\frac{\delta(1-s)^{\alpha-4}}{(\gamma-1)(\delta-1) \Gamma(\alpha-3)} \\
= & \frac{(\alpha+\delta-3)(1-s)^{\alpha-3}}{(\alpha-2)(\delta-1) \Gamma(\alpha-2)}+\frac{\delta(1-s)^{\alpha-4}}{(\gamma-1)(\delta-1) \Gamma(\alpha-3)} \stackrel{\Delta}{=} w_{2}(s) .
\end{aligned}
$$

(ii) If $0 \leq t<s<1$, then by an argument similar to (2.25), from (2.21), we have

$$
\begin{aligned}
-\frac{\partial G_{2}(t, s)}{\partial t} & =\frac{(1-s)^{\alpha-2}}{\Gamma(\alpha-1)}+\frac{(1-t)(1-s)^{\alpha-3}}{(\delta-1) \Gamma(\alpha-2)}+\frac{(1-t)[1+\delta+(\delta-1) t]}{2(\gamma-1)(\delta-1) \Gamma(\alpha-3)}(1-s)^{\alpha-4} \\
& \leq w_{2}(s)
\end{aligned}
$$

Summing up the above analysis (i)-(ii), and noting Step 2 of the proof as before, it follows that

$$
0<-\frac{\partial G(t, s)}{\partial t} \leq w_{2}(s) \text { for } s, t \in[0,1), s \neq t
$$

Step 4. It remains to show that

$$
-\frac{\partial G(t, s)}{\partial t} \geq \eta_{2} w_{2}(s) \quad \text { for } t \in\left[\frac{1}{6}, \frac{1}{3}\right], s \in[0,1) \text { with } s \neq t
$$

(i) If $0 \leq s<t<1$, then by (2.20) and the fact that $(1-s)^{\alpha-2}>(t-s)^{\alpha-2}$, we know that the relations

$$
\begin{aligned}
-\frac{\partial G_{1}}{\partial t} & >\frac{(1-t)(1-s)^{\alpha-3}}{(\delta-1) \Gamma(\alpha-2)}+\frac{(1-t)[1+\delta+(\delta-1) t]}{2(\gamma-1)(\delta-1) \Gamma(\alpha-3)}(1-s)^{\alpha-4} \\
& \geq \frac{2}{3} \frac{(1-s)^{\alpha-3}}{(\delta-1) \Gamma(\alpha-2)}+\frac{(1+\delta)(1-s)^{\alpha-4}}{3(\gamma-1)(\delta-1) \Gamma(\alpha-3)} \\
& =\frac{2(\alpha-2)}{3(\alpha+\delta-3)} \cdot \frac{(\alpha+\delta-3)(1-s)^{\alpha-3}}{(\alpha-2)(\delta-1) \Gamma(\alpha-2)}+\frac{1+\delta}{3 \delta} \cdot \frac{\delta(1-s)^{\alpha-4}}{(\gamma-1)(\delta-1) \Gamma(\alpha-3)} \\
& \geq \eta_{2} w_{2}(s)
\end{aligned}
$$

hold for $t \in\left[\frac{1}{6}, \frac{1}{3}\right], s \in[0,1)$ with $s<t$, where $\eta_{2}=\min \left\{\frac{2(\alpha-2)}{3(\alpha+\delta-3)}, \frac{1+\delta}{3 \delta}\right\}$, and

$$
w_{2}(s)=\frac{(\alpha+\delta-3)(1-s)^{\alpha-3}}{(\alpha-2)(\delta-1) \Gamma(\alpha-2)}+\frac{\delta(1-s)^{\alpha-4}}{(\gamma-1)(\delta-1) \Gamma(\alpha-3)}, \quad s \in[0,1)
$$


Similarly, we can obtain that

$$
-\frac{\partial G_{2}}{\partial t} \geq \eta_{2} w_{2}(s) \quad \text { for } t \in\left[\frac{1}{6}, \frac{1}{3}\right] \text { with } t<s<1
$$

The proof is complete.

Now, we introduce a cone as follows:

$$
\begin{gathered}
P=\left\{u \in C^{1}[I, R]: u(t) \geq 0, u^{\prime}(t) \leq 0, t \in I ; u(1)=0 ;\right. \\
\left.u(t) \geq \eta_{1}\|u\|_{0},-u^{\prime}(t) \geq \eta_{2}\left\|u^{\prime}\right\|_{0}, t \in\left[\frac{1}{6}, \frac{1}{3}\right]\right\} .
\end{gathered}
$$

It is easy to check that the above set $P$ is a cone in the space $C^{1}[I, R]$, which will be used in the sequel.

We define an operator $T$ on $P$ as follows:

$$
T u=\int_{0}^{1} G(t, s) f\left(s, u(s), u^{\prime}(s)\right) d s, \quad u \in P .
$$

Obviously, under the assumption $\left(H_{1}\right)-\left(H_{2}\right)$, the operator $T$ is well defined. Moreover,

$$
(T u)^{\prime}(t)=\int_{0}^{1} G_{t}(t, s) f\left(s, u(s), u^{\prime}(s)\right) d s, \quad t \in I, u \in P
$$

where $G_{t}(t, s)=\frac{\partial}{\partial t} G_{1}(t, s), 0 \leq s<t<1, G_{t}(t, s)=\frac{\partial}{\partial t} G_{2}(t, s), 0 \leq t<s<1$, and $\frac{\partial}{\partial t} G_{1}(t, s)$, $\frac{\partial}{\partial t} G_{2}(t, s)$ are given by (2.20)-(2.21), respectively.

A function $u \in C^{3}[0,1]$ is a positive solution of BVP (1.1)-(1.2) if $u(t) \geq 0, t \in I, u \neq 0$, and $u$ satisfies BVP (1.1)-(1.2).

By Lemma 2.3, it is easy to know that a function $u \in C^{3}[0,1]$ is a positive solution of BVP (1.1)-(1.2) iff $u \in P$ is a nonzero fixed point of $T$. So, we can focus on seeking the existence of a nonzero fixed point of $T$ in $P$.

Finally, for the remainder of this section, we give the following two theorems, which are fundamental in the proof of our main results.

Let $X$ be a Banach space, and let $P \subset X$ be a cone. Suppose that the functions $\alpha, \beta$ satisfy the following condition:

(D) $\alpha, \beta: X \rightarrow \mathbb{R}_{+}$are continuous convex functionals satisfying $\alpha(\lambda u)=|\lambda| \alpha(u)$, $\beta(\lambda u)=|\lambda|(u)$ for $u \in X, \lambda \in R ;\|u\| \leq k \max \{\alpha(u), \beta(u)\}$ for $u \in X$, and $\alpha\left(u_{1}\right) \leq \alpha\left(u_{2}\right)$ for $u_{1}, u_{2} \in P$ with $u_{1} \leq u_{2}$, where $k>0$ is a constant.

Lemma 2.5 [31] Assume that $r_{1}, r_{2}, L$ are constants with $r_{2}>r_{1}>0, L>0$, and

$$
\Omega_{i}=\left\{u \in X: \alpha(u)<r_{i}, \beta(u)<L\right\}, \quad i=1,2 \text { are two bounded open sets in } X
$$

Set $D_{i}=\left\{u \in X: \alpha(u)=r_{i}\right\}$. Suppose that $T: P \rightarrow P$ is a completely continuous operator satisfying

$\left(C_{1}\right) \alpha(T u)<r_{1}, u \in D_{1} \cap P ; \alpha(T u)>r_{2}, u \in D_{2} \cap P$; 
$\left(C_{2}\right) \beta(T u)<L, u \in P$;

$\left(C_{3}\right)$ there is a $p \in\left(\Omega_{2} \cap P\right) \backslash\{0\}$ such that $\alpha(p) \neq 0$ and $\alpha(u+\lambda p) \geq \alpha u$ for all $u \in P$ and $\lambda \geq 0$.

Then $T$ has at least one fixed point in $\left(\Omega_{2} \backslash \bar{\Omega}_{1}\right) \cap P$.

Lemma 2.6 [32] Assume that $\Omega_{1}, \Omega_{2}$ are two open subsets of $X$ with $0 \in \Omega_{1} \subset \bar{\Omega}_{1} \subset \Omega_{2}$, and let $T: P \rightarrow P$ be a completely continuous operator such that either

(i) $\|T u\| \leq\|u\|, u \in P \cap \partial \Omega_{1} ;\|T u\| \geq\|u\|, u \in P \cap \partial \Omega_{2}$, or

(ii) $\|T u\| \geq\|u\|, u \in P \cap \partial \Omega_{1} ;\|T u\| \leq\|u\|, u \in P \cap \partial \Omega_{2}$.

Then $T$ has a fixed point in $P \cap\left(\bar{\Omega}_{1} \backslash \Omega_{1}\right)$.

\section{Main results}

We first prove the following lemma to obtain our main results.

Lemma 3.1 Suppose that $\left(H_{1}\right)-\left(H_{2}\right)$ hold. Then the operator $T$ defined by (2.26) maps $P$ into $P$, and $T$ is completely continuous.

Proof It is well known that the norms $\|u\|_{1}=\|u\|_{0}+\left\|u^{\prime}\right\|_{0}$ and $\|u\|_{2}=\max \left\{\|u\|_{0},\left\|u^{\prime}\right\|_{0}\right\}$ are equivalent on $C^{1}[0,1]$. So, we can consider that the Banach space $C^{1}[0,1]$ is equipped with the norm $\|u\|_{2}=\max \left\{\|u\|_{0},\left\|u^{\prime}\right\|_{0}\right\}$ in the following proof.

For any $u \in P$, in view of the conclusion (1)-(2) of Lemma 2.4 and the hypotheses $\left(H_{1}\right)$ $\left(H_{2}\right)$, it is easy to see that $T u \in C^{1}(I, R),(T u)(t) \geq 0, t \in I$, and $(T u)^{\prime}(t) \leq 0, t \in I$ observing (2.26)-(2.27). Moreover, the conclusion (3) of Lemma 2.4 implies that

$$
\begin{aligned}
(T u)(t) & =\int_{0}^{1} G(t, s) f\left(s, u(s), u^{\prime}(s)\right) d s \\
& \leq \int_{0}^{1} w_{1}(s) f\left(s, u(s), u^{\prime}(s)\right) d s, \quad t \in I,
\end{aligned}
$$

and

$$
\begin{aligned}
(T u)(t) & =\int_{0}^{1} G(t, s) f\left(s, u(s), u^{\prime}(s)\right) d s \\
& \geq \eta_{1} \int_{0}^{1} w_{1}(s) f\left(s, u(s), u^{\prime}(s)\right) d s
\end{aligned}
$$

for $t \in\left[\frac{1}{6}, \frac{1}{3}\right]$.

From (3.1)-(3.2), it follows that $(T u)(t) \geq \eta_{1}(T u)(\tau), t \in\left[\frac{1}{6}, \frac{1}{3}\right], \tau \in I$. Thus,

$$
(T u)(t) \geq \eta_{1}\|T u\|_{0}, \quad t \in\left[\frac{1}{6}, \frac{1}{3}\right] .
$$

Similarly, we can obtain

$$
-(T u)^{\prime}(t) \geq \eta_{2}\left\|(T u)^{\prime}\right\|, \quad t \in\left[\frac{1}{6}, \frac{1}{3}\right] .
$$

In addition, $(T u)(1)=0$. Thus, $T: P \rightarrow P$. 
Now, we show that the operator $T$ is compact on $P$.

In fact, let $U$ be an arbitrary bounded set in $P$. Then there exists a positive number $L$ such that $\|u\|_{2} \leq L$ for all $u \in U$, and so $\exists M>0$ such that $0 \leq f\left(t, u(s), u^{\prime}(s)\right) \leq M, t \in I$ for all $u \in U$.

In terms of Lemma 2.4, it follows from (2.26)-(2.27) that

$$
\begin{aligned}
0 & \leq(T u)(t)=\int_{0}^{1} G(t, s) f\left(s, u(s), u^{\prime}(s)\right) d s \\
& \leq M \int_{0}^{1} w_{1}(s) d s, \quad t \in I, \\
0 & \leq(-T u)^{\prime}(t)=\int_{0}^{1}\left(-G_{t}(t, s) f\left(s, u(s), u^{\prime}(s)\right) d s\right. \\
& \leq M \int_{0}^{1} w_{2}(s) d s, \quad t \in I .
\end{aligned}
$$

Because the functions $w_{1}$ and $w_{2}$ are integrable on $I$, the formulae (3.3)-(3.4) yield that $\|T u\|_{0} \leq M_{0},\left\|(T u)^{\prime}\right\|_{0} \leq M_{0}$, where $M_{0}=M \cdot \max \left\{\int_{0}^{1} w_{1}(s) d s, \int_{0}^{1} w_{2}(s) d s\right\}$. So, $\|T u\|_{1} \leq M_{0}$. That is, $T U$ is uniformly bounded.

On the other hand, for any $t_{1}, t_{2} \in I$ with $t_{1}<t_{2}$, by setting $h=f\left(t, u, u^{\prime}\right)$, the formula (2.26) implies that

$$
\begin{aligned}
\left|(T u)\left(t_{2}\right)-(T u)\left(t_{1}\right)\right|= & \mid \int_{0}^{t_{1}}\left(G_{1}\left(t_{2}, s\right)-G_{1}\left(t_{1}, s\right)\right) h(s) d s \\
& +\int_{t_{1}}^{t_{2}}\left(G_{1}\left(t_{2}, s\right)-G_{2}\left(t_{1}, s\right)\right) h(s) d s \\
& +\int_{t_{2}}^{1}\left(G_{2}\left(t_{2}, s\right)-G_{2}\left(t_{1}, s\right)\right) h(s) d s \mid \\
\leq & M \int_{0}^{t_{1}}\left|G_{1}\left(t_{2}, s\right)-G_{1}\left(t_{1}, s\right)\right| d s+2 M \int_{t_{1}}^{t_{2}} w_{1}(s) d s \\
& +M \int_{t_{2}}^{1}\left|G_{2}\left(t_{2}, s\right)-G_{2}\left(t_{1}, s\right)\right| d s .
\end{aligned}
$$

According to (2.4)-(2.5) and by applying the mean value theorem, we have

$$
\left|G_{1}\left(t_{2}, s\right)-G_{1}\left(t_{1}, s\right)\right| \leq\left[\frac{2}{\Gamma(\alpha-1)}+\frac{1}{(\delta-1) \Gamma(\alpha-2)}+\frac{\delta(1-s)^{\alpha-4}}{(\gamma-1)(\delta-1) \Gamma(\alpha-3)}\right]\left|t_{2}-t_{1}\right|,
$$

and so

$$
\int_{0}^{t_{1}}\left|G_{1}\left(t_{2}, s\right)-G_{1}\left(t_{1}, s\right)\right| d s \leq N_{1}\left|t_{2}-t_{1}\right|
$$

where $N_{1}=\frac{2}{\Gamma(\alpha-1)}+\frac{1}{(\delta-1) \Gamma(\alpha-2)}+\frac{\delta}{(\gamma-1)(\delta-1) \Gamma(\alpha-2)}$.

Similarly, there is another constant $N_{2}$ such that

$$
\int_{t_{2}}^{1}\left|G_{2}\left(t_{2}, s\right)-G_{2}\left(t_{1}, s\right)\right| d s \leq N_{2}\left|t_{1}-t_{2}\right| .
$$


Again, because the function $w_{1}$ is integrable on $I$, the absolute continuity of integral of $w_{1}$ on $\left[t_{1}, t_{2}\right]$ ensures that there exists a constant $N_{3}$ such that

$$
\int_{t_{1}}^{t_{2}} w_{1}(s) d s<N_{3}\left(t_{2}-t_{1}\right)
$$

So, (3.5) together with (3.6)-(3.8) implies that there exists a constant $N$ such that the inequality

$$
\left|(T u)\left(t_{2}\right)-(T u)\left(t_{1}\right)\right| \leq N\left(t_{2}-t_{1}\right)
$$

holds for any $u \in U$ and $t_{1}, t_{2} \in I$ with $t_{1}<t_{2}$. That is, the set $T U$ is equicontinuous.

Similarly, we can deduce that the set $\left\{(T u)^{\prime} \mid u \in U\right\}$ is also equicontinuous in terms of (2.27).

So, as a consequence of the Arzelà-Ascoli theorem, we have that $T U$ is a compact set.

Now, we come to prove the operator $T$ is continuous on $P$.

Let $\left\{u_{n}\right\}$ be an arbitrary sequence in $P$ with $u_{n} \rightarrow u_{0} \in P$. Then there exists an $L>0$ such that

$$
u_{n}(t) \in[0, L], \quad u_{n}^{\prime}(t) \in[-L, 0], \quad t \in I, n=0,1,2, \ldots
$$

According to the uniform continuity of $f$ on $I \times[0, L] \times[-L, 0]$, for an arbitrary number $\varepsilon>0$, there is a number $N \geq 1$ such that

$$
\left|f\left(t, u_{n}(t), u_{n}^{\prime}(t)\right)-f\left(t, u_{0}(t), u_{0}^{\prime}(t)\right)\right|<\varepsilon
$$

for all $t \in I$, whenever $n \geq N$.

Thus, in view of Lemma 2.4, from (2.26)-(2.27) and (3.9), it follows that

$$
\left|\left(T u_{n}\right)(t)-\left(T u_{0}\right)(t)\right| \leq \varepsilon \int_{0}^{1} w_{1}(s) d s, \quad t \in I
$$

and

$$
\left|\left(T u_{n}\right)^{\prime}(t)-(T u)^{\prime}(t)\right| \leq \varepsilon \int_{0}^{1} w_{2}(s) d s, \quad t \in I
$$

whenever $n \geq N$. That is, $T$ is continuous on $P$.

We are now in a position to state and prove the first theorem in the article. Let constants $k_{1}, k_{2}$ satisfy $k_{1} \in\left(0,\left(\int_{0}^{1} w_{2}(s) d s\right)^{-1}\right), k_{2}>\left(\eta_{2} \int_{\frac{1}{6}}^{\frac{1}{3}} w_{2}(s) d s\right)^{-1}$.

Theorem 3.1 Suppose that $\left(H_{1}\right)-\left(H_{2}\right)$ hold. In addition, there are two constants $r_{1}, r_{2}$ with $\frac{1}{6} \eta_{1} \eta_{2} r_{2}>r_{1}>0$ such that $f$ satisfies the following condition:

( $\left.H_{3}\right) f(t, x, y) \leq k_{1} r_{1}$, for $(t, x, y) \in I \times\left[0, r_{1}\right] \times\left[-r_{1}, 0\right]$;

$$
f(t, x, y) \geq k_{2} r_{2}, \text { for }(t, x, y) \in I \times\left[\frac{1}{6} \eta_{1} \eta_{2} r_{2}, r_{2}\right] \times\left[-r_{2},-\eta_{2} r_{2}\right] .
$$

Then BVP (1.1)-(1.2) has at least one positive solution $u$ satisfying $r_{1}<\max _{t \in I}\left|u^{\prime}(t)\right|<r_{2}$ and $\max _{t \in I}|u(t)|<r_{2}$. 
Proof We already know that $T: P \rightarrow P$ is completely continuous by Lemma 3.1.

Let $\alpha(u)=\max _{t \in I}\left|u^{\prime}(t)\right|, \beta(u)=\max _{t \in I}|u(t)|$ for $u \in P$. It is easy to verify that the functions $\alpha, \beta$ satisfy the condition (D).

Choose a constant $L$ large enough so that $L>\max \left\{M \int_{0}^{1} w_{1}(s) d s, r_{2}\right\}$, where $M=$ $\max _{(t, x, y) \in I \times J \times \Delta} f(t, x, y)$, and $J=\left[0, r_{2}\right], \Delta=\left[-r_{2}, 0\right]$. Set $D_{i}=\left\{u \in C^{1}(I, R): \alpha(u)=r_{i}\right\}$, $\Omega_{i}=\left\{u \in C^{1}(I, R): \alpha(u)<r_{i}, \beta(u)<L\right\}, i=1,2$. Define the function $\bar{f}$ on $I \times \mathbb{R}_{+} \times \mathbb{R}_{-}$as $\bar{f}(t, x, y)=f(t, \phi(x), \varphi(y)),(t, x, y) \in I \times \mathbb{R}_{+} \times \mathbb{R}_{-}$, where $\phi(x)=\min \left\{x, r_{2}\right\}, \varphi(y)=\max \left\{y,-r_{2}\right\}$.

Consider the following ancillary BVP:

$$
\left\{\begin{array}{l}
{ }^{C} D_{0+}^{\alpha} x(t)=\bar{f}\left(t, x(t), x^{\prime}(t)\right), \quad t \in(0.1), \\
x(1)=x^{\prime}(1)=0, \quad \delta x^{\prime \prime}(0)=x^{\prime \prime}(1), \quad \gamma x^{\prime \prime \prime}(0)=x^{\prime \prime \prime}(1) .
\end{array}\right.
$$

Obviously, the function $\bar{f}$ is continuous on $I \times \mathbb{R}_{+} \times \mathbb{R}_{-}$according to the continuity of $f$. Thus, by an argument similar to that in Lemma 3.1, the operator $\bar{T}$ given by $\bar{T} u=$ $\int_{0}^{1} G(t, s) \bar{f}\left(s, u(s), u^{\prime}(s)\right) d s$ is also completely continuous on $P$ and maps $P$ into $P$.

We will prove that $T$ has at least one nonzero fixed point in $P$ by applying Lemma 2.5 . The approach is divided into four steps.

Step 1 . We first show that

$$
\|u\|_{0} \leq\left\|u^{\prime}\right\|_{0}, \quad u(t) \geq \frac{1}{6} \eta_{1} \eta_{2}\left\|u^{\prime}\right\|_{0}, \quad t \in\left[\frac{1}{6}, \frac{1}{3}\right] \quad \text { for any } u \in P .
$$

In fact, for any $u \in P$, owing to the condition $u(1)=0$, we have that $u(t)=-\int_{t}^{1} u^{\prime}(s) d s$, $t \in I$, and so $\|u\|_{0} \leq\left\|u^{\prime}\right\|_{0}$.

On the other hand, applying the mean value theorem, for any $u \in P$, we have that $\|u\|_{0} \geq$ $\left|u\left(\frac{1}{3}\right)-u\left(\frac{1}{6}\right)\right|=\frac{1}{6}\left|u^{\prime}(\xi)\right|$ for some $\xi \in\left(\frac{1}{6}, \frac{1}{3}\right)$. Therefore, we have that $\|u\|_{0} \geq \frac{1}{6} \eta_{2}\left\|u^{\prime}\right\|_{0}$ from the fact that $-u^{\prime}(\xi) \geq \eta_{2}\left\|u^{\prime}\right\|_{0}, \xi \in\left[\frac{1}{6}, \frac{1}{3}\right]$ because $u \in P$. So,

$$
u(t) \geq \eta_{1}\|u\|_{0} \geq \frac{1}{6} \eta_{1} \eta_{2}\left\|u^{\prime}\right\|_{0}, \quad t \in\left[\frac{1}{6}, \frac{1}{3}\right]
$$

keeping in mind that $u(t) \geq \eta_{1}\|u\|_{0}, t \in\left[\frac{1}{6}, \frac{1}{3}\right]$ for $u \in P$.

Step 2 . Now, we come to verify that the conditions corresponding to $\left(C_{1}\right)$ in Lemma 2.5 hold.

For any $u \in P$ with $\alpha(u)=r_{1}$, we have that $u(t) \geq 0, u^{\prime}(t) \leq 0, t \in I$, and $\left\|u^{\prime}\right\|_{0}=r_{1}$. Thus, in view of (3.11), we have that $0 \leq u(t) \leq r_{1},-r_{1} \leq u^{\prime}(t) \leq 0, t \in I$. So, according to $\left(H_{3}\right)$, we have

$$
\bar{f}\left(t, u(t), u^{\prime}(t)\right)=f\left(t, u(t), u^{\prime}(t)\right) \leq k_{1} r_{1}, \quad t \in I
$$

Thus, from (2.27) and Lemma 2.4, it follows that

$$
\begin{aligned}
-(\bar{T} u)^{\prime}(t) & =-\int_{0}^{1} G_{t}(t, s) f\left(s, u(s), u^{\prime}(s)\right) d s \\
& \leq k_{1} r_{1} \int_{0}^{1}\left(-G_{t}(t, s)\right) d s \leq k_{1} r_{1} \int_{0}^{1} w_{2}(s) d s, \quad t \in I .
\end{aligned}
$$


Thus, $\max _{t \in I}\left|(\bar{T} u)^{\prime}(t)\right| \leq k_{1} r_{1} \int_{0}^{1} w_{2}(s) d s<r_{1}$, noting that the assumption $k_{1}<$ $\left(\int_{0}^{1} w_{2}(s) d s\right)^{-1}$. That is, $\alpha(T u)<r_{1}$.

For any $u \in P$ with $\alpha(u)=r_{2}$, then $u(t) \geq 0, u^{\prime}(t) \leq 0, t \in I$, and $\max _{t \in I}\left|u^{\prime}(t)\right|=r_{2}$. Thus, from (3.11), we obtain

$$
\frac{1}{6} \eta_{1} \eta_{2} r_{2} \leq u(t) \leq r_{2}, \quad-r_{2} \leq u^{\prime}(t) \leq-\eta_{2} r_{2}, \quad t \in\left[\frac{1}{6}, \frac{1}{3}\right]
$$

and so

$$
\bar{f}\left(t, u(t), u^{\prime}(t)\right)=f\left(t, u(t), u^{\prime}(t)\right) \geq k_{2} r_{2}, \quad t \in\left[\frac{1}{6}, \frac{1}{3}\right],
$$

from the condition $\left(H_{3}\right)$. Therefore, in view of Lemma 2.4, we have

$$
\begin{aligned}
-(\bar{T} u)^{\prime}(t) & =-\int_{0}^{1} G_{t}(t, s) f\left(s, u(s), u^{\prime}(s)\right) d s \\
& \geq-\int_{\frac{1}{6}}^{\frac{1}{3}} G_{t}(t, s) f\left(s, u(s), u^{\prime}(s)\right) d s \\
& \geq k_{2} r_{2} \int_{\frac{1}{6}}^{\frac{1}{3}}\left(-G_{t}(t, s)\right) d s \geq k_{2} r_{2} \eta_{2} \int_{\frac{1}{6}}^{\frac{1}{3}} w_{2}(s) d s, \quad t \in I .
\end{aligned}
$$

Thus, $\max _{t \in I}\left|T^{\prime} u(t)\right| \geq k_{2} \eta_{2} r_{2} \int_{\frac{1}{6}}^{\frac{1}{3}} w_{2}(s) d s>r_{2}$, noting that $k_{2}>\left(\eta_{2} \int_{\frac{1}{6}}^{\frac{1}{3}} w_{2}(s) d s\right)^{-1}$. That is, $\alpha(T u)>r_{2}$.

Step 3. We verify that the conditions corresponding to $\left(C_{2}\right)$ in Lemma 2.5 hold.

For any $u \in P$, owing to the fact that $0 \leq \phi(u(t)) \leq r_{2},-r_{2} \leq \varphi\left(u^{\prime}(t)\right) \leq 0$, from the meaning of $M$, we have immediately that

$$
\bar{f}\left(t, u(t), u^{\prime}(t)\right)=f\left(t, \phi(u(t)), \varphi\left(u^{\prime}(t)\right)\right) \leq M, \quad t \in I .
$$

Thus,

$$
\begin{aligned}
(\bar{T} u)(t) & =\int_{0}^{1} G(t, s) \bar{f}\left(s, u(s), u^{\prime}(s)\right) d s \\
& \leq M \int_{0}^{1} G(t, s) d s \leq M \int_{0}^{1} w_{1}(s) d s, \quad t \in I .
\end{aligned}
$$

Hence, $\|(\bar{T} u)\|_{0} \leq M \int_{0}^{1} w_{1}(s) d s$, and so $\|(\bar{T} u)\|_{0}<L$ from the choice of $L$. Thus, $\beta(\bar{T} u)<L$. Step 4. Finally, take $p=\sigma \int_{0}^{1} G(t, s) d s$ with $0<\sigma<r_{2}\left(\int_{0}^{1} w_{2}(s) d s\right)^{-1}$. Then, by an argument similar to that in Lemma 3.1, we can know that $p \in P$. Moreover, $0 \leq-p^{\prime}(t)=$ $\sigma \int_{0}^{1}\left(-G_{t}(t, s)\right) d s \leq \sigma \int_{0}^{1} w_{2}(s) d s<r_{2}, t \in I$, and $0 \leq p(t)<r_{2}, t \in I$ from (3.11). Thus, $p \in\left(\Omega_{2} \cap P\right) \backslash\{0\}, \alpha(p) \neq 0$. Again,

$$
\begin{aligned}
\alpha(u+\lambda p) & =\max _{t \in I}\left|u^{\prime}+\lambda p^{\prime}(t)\right|=\max \left(-u^{\prime}(t)+\lambda\left(-p^{\prime}(t)\right)\right. \\
& \geq \lambda \max \left(-u^{\prime}(t)\right)=\lambda \alpha(u)
\end{aligned}
$$

for any $u \in P, \lambda \geq 0$. 
So, the conditions corresponding to $\left(C_{3}\right)$ in Lemma 2.5 hold.

Summing up the above steps 1-4 and applying Lemma 2.5, we obtain that BVP (3.10) has at least one positive solution $u \in\left(\Omega_{2} \backslash \bar{\Omega}_{1}\right) \cap P$. That is, $r_{1}<\left\|u^{\prime}\right\|_{0}<r_{2}$. $\|u\|_{0}<L$, and so $\|u\|_{0}<r_{2}$ from the fact that $r_{2}<L$ and $\|u\|_{0} \leq\left\|u^{\prime}\right\|_{0}$ by (3.13). Thus, $0 \leq u(t)<r_{2}, t \in I$, $0 \leq-u^{\prime}(t)<r_{2}, t \in I$, and $u^{\prime} \neq 0$. Hence, $\bar{f}\left(t, u(t), u^{\prime}(t)\right)=f\left(t, u(t), u^{\prime}(t)\right), t \in I$, and so $u$ is a positive solution of BVP (1.1)-(1.2). The proof is complete.

Now, we state another theorem in this paper. Let us begin with introducing some notations.

Let $B=\mathbb{R}_{+} \times \mathbb{R}_{-}$. Denote $f_{\infty}=\liminf _{x+|y| \rightarrow \infty} \min _{t \in I} \frac{f(t, x, y)}{x+|y|}$ with $(x, y) \in B$, and $f^{0}=$ $\lim \sup _{x+|y| \rightarrow 0} \max _{t \in I} \frac{f(t, x, y)}{x+|y|}$ with $(x, y) \in B$. Put $\lambda_{1}=\left(\eta_{0} \mu_{1}\right)^{-1}, \lambda_{2}=\mu_{2}^{-1}$, where $\mu_{1}=$ $\int_{\frac{1}{6}}^{\frac{1}{3}}\left[G(0, s)-G_{t}(0, s)\right] d s, \mu_{2}=\int_{0}^{1}\left(w_{1}(s)+w_{2}(s)\right) d s, \eta_{0}=\min \left\{\eta_{1}, \eta_{2}\right\}$, and $\eta_{i}(i=1,2)$ are given in Lemma 2.4 .

Theorem 3.2 Assume that $\left(H_{1}\right)-\left(H_{2}\right)$ hold. If $f_{\infty}>\lambda_{1}, f^{0}<\lambda_{2}$, then BVP (1.1)-(1.2) has at least one positive solution.

Proof As described in the proof of Theorem 3.1, $T: P \rightarrow P$ is completely continuous. Again, from $f_{\infty}>\lambda_{1}$, it follows that there exists an $R_{1}>0$ such that

$$
f(t, x, y)>\lambda_{1}(x+|y|), \quad t \in I
$$

holds when $x+|y| \geq R_{1}$ with $x \geq 0, y \leq 0$.

Take $R \geq \eta_{0}^{-1} R_{1}$. Set $\Omega_{R}=\left\{u \in C^{1}[0,1]:\|u\|_{1}<R\right\}$. Now, we show that the following relation holds:

$$
\|T u\|_{1} \geq\|u\|_{1}, \quad \forall u \in \partial \Omega_{R} \cap P .
$$

In fact, for any $u \in \partial \Omega_{R} \cap P$, we have that $u \in P$ with $\|u\|_{1}=R$. Then

$$
\begin{aligned}
& u(t) \geq 0, \quad u^{\prime}(t) \leq 0, \quad t \in I \\
& u(t) \geq \eta_{1}\|u\|_{0}, \quad-u^{\prime}(t) \geq \eta_{2}\left\|u^{\prime}\right\|_{0}, \quad t \in\left[\frac{1}{6}, \frac{1}{3}\right] .
\end{aligned}
$$

Thus,

$$
\begin{aligned}
u(t)+\left|u^{\prime}(t)\right| & \geq \eta_{1}\|u\|_{0}+\eta_{2}\left\|u^{\prime}\right\|_{0} \\
& \geq \eta_{0}\left(\|u\|_{0}+\left\|u^{\prime}\right\|_{0}\right)=\eta_{0} R \geq R_{1}, \quad \text { for } t \in\left[\frac{1}{6}, \frac{1}{3}\right] .
\end{aligned}
$$

Thus, from (3.12), (3.14), it follows that

$$
f\left(t, u(t), u^{\prime}(t)\right)>\lambda_{1} \eta_{0} R, \quad t \in\left[\frac{1}{6}, \frac{1}{3}\right] .
$$


Hence, (2.26) together with (3.15) implies that

$$
\begin{aligned}
(T u)(t) & =\int_{0}^{1} G(t, s) f\left(s, u(s), u^{\prime}(s)\right) d s \\
& \geq \int_{\frac{1}{6}}^{\frac{1}{3}} G(t, s) f\left(s, u(s), u^{\prime}(s)\right) d s \\
& \geq \lambda_{1} \eta_{0} R \int_{\frac{1}{6}}^{\frac{1}{3}} G(t, s) d s, \quad t \in I .
\end{aligned}
$$

So,

$$
\|T u\|_{0} \geq(T u)(0) \geq \lambda_{1} \eta_{0} R \int_{\frac{1}{6}}^{\frac{1}{3}} G(0, s) d s .
$$

Similarly, we can obtain

$$
\left\|(T u)^{\prime}\right\|_{0} \geq-(T u)^{\prime}(0) \geq \lambda_{1} \eta_{0} R \int_{\frac{1}{6}}^{\frac{1}{3}}\left[-G_{t}(0, s)\right] d s .
$$

Therefore, noting that $\lambda_{1}=\left(\eta_{0} \mu_{1}\right)^{-1}$, from (3.16)-(3.17), we have

$$
\begin{aligned}
\|T u\|_{1} & =\|T u\|_{0}+\left\|(T u)^{\prime}\right\|_{0} \\
& \geq \lambda_{1} \eta_{0} R \int_{\frac{1}{6}}^{\frac{1}{3}}\left[G(0, s)-G_{t}(0, s)\right] d s=\lambda_{1} \eta_{0} \mu_{1} R=R=\|u\|_{1} .
\end{aligned}
$$

So, the relation (3.13) holds.

Now, from $f^{0}<\lambda_{2}$, it follows that there exists an $r_{2}>0$ such that

$$
f(t, x, y)<\lambda_{2}(x+|y|), \quad t \in I
$$

whenever $x+|y| \leq r_{2}$ with $x \geq 0, y \leq 0$.

Take $0<r<\min \left\{r_{2}, R\right\}$. Set $\Omega_{r}=\left\{u \in C^{1}[0,1]:\|u\|_{1}<r\right\}$. We prove the following relation holds:

$$
\|T u\|_{1} \leq\|u\|_{1}, \quad \forall u \in \partial \Omega_{r} \cap P .
$$

In fact, for any $u \in \partial \Omega_{r} \cap P$, we have $u \in P$ and $\|u\|_{1}=r$. Thus, $u(t) \geq 0, u^{\prime}(t) \leq 0, t \in I$, and $u(t)+\left|u^{\prime}(t)\right| \leq r, t \in I$. So, by (3.18), it follows that

$$
f\left(t, u(t), u^{\prime}(t)\right)<\lambda_{2}\left(u(t)+\left|u^{\prime}(t)\right|\right) \leq \lambda_{2} r
$$

Therefore, from (2.26) and in view of Lemma 2.4, we have

$$
\begin{aligned}
(T u)(t) & =\int_{0}^{1} G(t, s) f\left(s, u(s), u^{\prime}(s)\right) d s \\
& \leq \lambda_{2} r \int_{0}^{1} G(t, s) d s \leq \lambda_{2} r \int_{0}^{1} w_{1}(s) d s, \quad t \in I .
\end{aligned}
$$


So,

$$
\|T u\|_{0} \leq \lambda_{2} r \int_{0}^{1} w_{1}(s) d s
$$

Similarly, we can obtain

$$
\left\|(T u)^{\prime}\right\|_{0} \leq \lambda_{2} r \int_{0}^{1} w_{2}(s) d s
$$

Consequently, noting that $\lambda_{2}=\mu_{2}^{-1}$, from (3.21)-(3.22), it follows that

$$
\begin{aligned}
\|T u\|_{1} & =\|T u\|_{0}+\left\|(T u)^{\prime}\right\|_{0} \\
& \leq \lambda_{2} r \int_{0}^{1}\left(w_{1}(s)+w_{2}(s)\right) d s=r \lambda_{2} \mu_{2}=r=\|u\|_{1} .
\end{aligned}
$$

So, the relation (3.19) holds.

Summing up (3.13) and (3.19), applying Lemma 2.6, the operator $T$ has at least one fixed point $u \in\left(\bar{\Omega}_{2} \backslash \Omega_{1}\right) \cap P$. Thus $u$ is a positive solution of BVP (1.1)-(1.2). The proof is complete.

Example 3.1 Consider the following BVP:

$$
\left\{\begin{array}{l}
{ }^{C} D_{0+}^{\alpha} x(t)=f\left(t, x(t), x^{\prime}(t)\right), \quad t \in(0,1), \\
x(1)=x^{\prime}(1)=0, \quad \delta x^{\prime \prime}(0)=x^{\prime \prime}(1), \quad \gamma x^{\prime \prime \prime}(0)=x^{\prime \prime \prime}(1),
\end{array}\right.
$$

where $3<\alpha \leq 4, \gamma>1, \delta>1$, and $f$ is given by

$$
f(t, x, y)=e^{t}\left[a_{1}(\sin \sqrt{x})^{6}+a_{2}(\sqrt{-y})^{4}\right], \quad(t, x, y) \in I \times \mathbb{R}_{+} \times \mathbb{R}_{-}
$$

where constants $a_{1}, a_{2}$ are two positive numbers. Then BVP (3.23) has at least one positive solution.

In fact, assume that the notations $k_{1}, k_{2}, \eta_{1}$, and $\eta_{2}$ are described in Theorem 3.1. Take $0<r_{1}<\min \left\{1, \frac{k_{1}}{e\left(a_{1}+a_{2}\right)}\right\}, r_{2}>\max \left\{\frac{k_{2}}{a_{2} \eta_{2}^{2}}, \frac{6}{\eta_{1} \eta_{2}}\right\}$. Then the inequality

$$
f(t, x, y) \leq e\left[a_{1} x^{3}+a_{2} y^{2}\right] \leq e\left[a_{1}+a_{2}\right] r_{1}^{2} \leq k_{1} r_{1}
$$

holds for $(t, x, y) \in I \times\left[0, r_{1}\right] \times\left[-r_{1}, 0\right]$, and the inequality

$$
f(t, x, y) \geq a_{2} y^{2} \geq a_{2} \eta_{2}^{2} r_{2}^{2} \geq k_{2} r_{2}
$$

holds for $(t, x, y) \in I \times[0,+\infty) \times\left[-r_{2},-\eta_{2} r_{2}\right]$.

So, by Theorem 3.1, BVP (3.23) has at least one positive solution.

Example 3.2 Consider the following BVP:

$$
\left\{\begin{array}{l}
{ }^{C} D_{0+}^{\alpha} x(t)=f\left(t, x(t), x^{\prime}(t)\right), \quad t \in(0,1), \\
x(1)=x^{\prime}(1)=0, \quad \delta x^{\prime \prime}(0)=x^{\prime \prime}(1), \quad \gamma x^{\prime \prime \prime}(0)=x^{\prime \prime \prime}(1),
\end{array}\right.
$$


where $3<\alpha \leq 4, \gamma>1, \delta>1$, and $f$ is given by

$$
f(t, x, y)=e^{t}\left[a_{1}(x+|y|)^{\beta}+a_{2}(\sin x y)^{2}\right], \quad(t, x, y) \in I \times \mathbb{R}_{+} \times \mathbb{R}_{-},
$$

where constants $a_{1}, a_{2}$ are two positive numbers and a constant $\beta>1$. Then BVP (3.24) has at least one positive solution.

In fact, observing that $f_{\infty}=+\infty, f^{0}=0$, the conclusion follows from Theorem 3.2.

\section{Competing interests}

The author declares that he has no competing interests.

\section{Acknowledgements}

The author sincerely thanks the anonymous referees for their valuable suggestions and comments which have greatly helped improve this article. Article is supported by the Natural Science Foundation of Hubei Provincial Education Department (D20102502).

\section{Received: 15 October 2012 Accepted: 15 April 2013 Published: 29 April 2013}

\section{References}

1. Glockle, WG, Nonnenmacher, TF: A fractional calculus approach of self-similar protein dynamics. Biophys. J. 68, 46-53 (1995)

2. Hilfer, R: Applications of Fractional Calculus in Physics. World Scientific, Singapore (2000)

3. Metzler, F, Schick, W, Kilian, HG, Nonnenmacher, TF: Relaxation in filled polymers: a fractional calculus approach. J. Chem. Phys. 103, 7180-7186 (1995)

4. Podlubny, I: Geometric and physical interpretation of fractional integration and fractional differentiation. Fract. Calc. Appl. Anal. 5, 367-386 (2002)

5. Podlubny, I: Fractional Differential Equations. Academic Press, San Diego (1999)

6. Kilbas, AA, Srivastava, HM, Trujillo, JJ: Theory and Applications of Fractional Differential Equations. North-Holland Mathematics Studies, vol. 204. Elsevier, Amsterdam (2006)

7. Lakshmikantham, V, Leela, S, Vasundhara, J: Theory of Fractional Dynamic Systems. Cambridge Academic Publishers, Cambridge (2009)

8. Samko, SG, Kilbas, AA, Marichev, IO: Fractional Integrals and Derivatives. Theory and Applications. Gordon \& Breach, Yverdon (1993)

9. Diethelm, K: The Analysis of Fractional Differential Equations. Springer, New York (2010)

10. Agarwal, RP, Benchohra, N, Hamani, S: A survey on existence result for boundary value problems of nonlinear fractional differential equations and inclusions. Acta Appl. Math. 109, 973-1033 (2010)

11. Agarwal, RP, O'Regan, D, Stanek, S: Positive solutions for Dirichlet problems of singular nonlinear fractional differential equations. J. Math. Anal. Appl. 371, 57-68 (2010)

12. Cabada, A, Wang, G: Positive solutions of nonlinear fractional differential equations with integral boundary value conditions. J. Math. Anal. Appl. 389, 403-411 (2012)

13. Ahmad, B, Nieto, JJ: Sequential fractional differential equations with three-point boundary conditions. Comput. Math Appl. 64, 3046-3052 (2012)

14. Xu, X, Jiang, D, Yuan, C: Multiple positive solutions for the boundary value problem of a nonlinear fractional differential equation. Nonlinear Anal. 71, 4676-4688 (2009)

15. Liang, S, Zhang, J: Positive solutions for boundary value problems of nonlinear fractional differential equation. Nonlinear Anal. 71, 5545-5550 (2009)

16. Agarwal, RP, Ahmad, B: Existence theory for anti-periodic boundary value problems of fractional differential equations and inclusions. Comput. Math. Appl. 62, 1200-1214 (2011)

17. Chai, G: Existence results for boundary value problems of nonlinear fractional differential equations. Comput. Math. Appl. 62, 2374-2382 (2011)

18. Chai, G: Positive solutions for boundary value problem of fractional differential equation with $p$-Laplacian operator. Bound. Value Probl. 2012, 18 (2012)

19. Zhai, C, Hao, M: Fixed point theorems for mixed monotone operators with perturbation and applications to fractional differential equation boundary value problems. Nonlinear Anal. 75, 2542-2551 (2012)

20. Guezane-Lakoud, A, Khaldi, R: Solvability of fractional boundary value problem with fractional integral condition. Nonlinear Anal. 75, 2692-2700 (2012)

21. Karthikeyan, K, Trujjllo, JJ: Existence and uniqueness results for fractional integrodifferential equations with boundary value conditions. Commun. Nonlinear Sci. Numer. Simul. 17, 4037-4043 (2012)

22. Abmad, B, Ntouyas, S: Existence results for nonlocal boundary value problems of fractional differential equations and inclusions with strip conditions. Bound. Value Probl. 2012, 55 (2012)

23. Goodrich, CS: On a fractional boundary value problem with fractional boundary conditions. Appl. Math. Lett. 25, 1101-1105 (2012)

24. Debbouche, A, Baleanu, D, Agarwal, RP: Nonlocal nonlinear integrodifferential equations of fractional orders. Bound. Value Probl. 2012, 78 (2012)

25. Zhang, L, Wang, G, Song, G: On mixed boundary value problem of impulsive semilinear evolution equations of fractional order. Bound. Value Probl. 2012, 17 (2012) 
26. Webb, JRL, Zima, M: Multiple positive solutions of resonant and non-resonant non-local four-order boundary value problem. Glasg. Math. J. 54, 225-240 (2012)

27. Chai, G: Existence of positive solutions for fourth-order boundary value problem with variable parameters. Nonlinear Anal. 66, 870-880 (2007)

28. Guo, Y, Yang, F, Liang, Y: Positive solutions for nonlocal fourth-order boundary value problems with all order derivatives. Bound. Value Probl. 2012, 29 (2012)

29. Chai, G: Positive solution of fourth-order integral boundary value problem with two parameters. Abstr. Appl. Anal. 2011, Article ID 859497 (2011)

30. Cai, J, Liu, G: Positive solutions for a class of fourth-order boundary value problems in Banach spaces. Abstr. Appl. Anal. 2011, Article ID 831730 (2011)

31. Guo, Y, Ge, W: Positive solutions for three-point boundary value problems with dependence on the first order derivative. J. Math. Anal. Appl. 290, 291-301 (2004)

32. Guo, D, Lakshimikantham, V: Nonlinear Problems in Abstract Cones. Academic Press, New York (1988)

doi:10.1186/1687-2770-2013-109

Cite this article as: Chai: Existence results of positive solutions for boundary value problems of fractional differential equations. Boundary Value Problems 2013 2013:109.

\section{Submit your manuscript to a SpringerOpen ${ }^{\circ}$ journal and benefit from:}

- Convenient online submission

- Rigorous peer review

- Immediate publication on acceptance

- Open access: articles freely available online

- High visibility within the field

- Retaining the copyright to your article 